Revista Destaques Acadêmicos, Lajeado, v. 8, n. 4, 2016. ISSN 2176-3070

DOI: http://dx.doi.org/10.22410/issn.2176-3070.v8i4a2016.1191

www.univates.br/revistas

\title{
INTERCONEXÕES ENTRE NÚMEROS COMPLEXOS E LUGARES GEOMÉTRICOS
}

\author{
Thiago Beirigo Lopes ${ }^{1}$, Leniedson Guedes dos Santos ${ }^{2}$
}

Resumo: A matemática normalmente tem seus conteúdos estudados de modo desconectados entre si, assim podendo passar a ideia que não há relação entre tais conteúdos. Ao se estudar Lugares Geométricos e Números Complexos é comum fazêlo de modo que não crie relações entre tais conhecimentos. Neste trabalho é explorada uma abordagem de Lugares Geométricos no plano complexo, utilizando os Números Complexos para definirmos mediatriz, círculo, elipse, hipérbole e parábola. Este trabalho se enquadra como básico puro, por se dedicar exclusivamente à ampliação do conhecimento.

Palavras-chave: Matemática. Números Complexos. Lugar Geométrico. Plano complexo.

\section{INTRODUÇÃO}

Como todo conhecimento científico, os conceitos matemáticos passam por um processo de desenvolvimento no qual incorpora mudanças, criando novas ferramentas ou métodos que permitem um incremento no conhecimento pré-estabelecido. Nessa evolução, também há criação de conexões entre conhecimentos matemáticos. Porém, no tocante ao estudo de matemática, seus conteúdos normalmente são estudados de modo separados, podendo assim dar a impressão de que não há relação entre tais conhecimentos. Portanto, é interessante mostrar as interconexões que são estabelecidas entre esses conhecimentos de modo a expandir a compreensão que quem os estuda.

Os conteúdos de Números Complexos e Lugares Geométricos são frequentemente vistos desassociados entre si. Segundo Lima et al. (2004), um número complexo é um número $z$ que pode ser expresso na forma

1 Doutorando em Ensino de Ciências e Matemática pela Universidade Federal de Mato Grosso (UFMT). Professor efetivo do Instituto Federal de Mato Grosso (IFMT). E-mail: thiagobeirigolopes@yahoo.com.br

2 Mestre em Matemática pela Universidade Federal do Tocantins (UFT). Professor assistente da Universidade Federal do Oeste da Bahia (UFOB). E-mail: leniedson@hotmail.com 
$z=x+y i$, onde $x$ e $y$ são números reais e $i$ significa a unidade imaginária. Tendo esta unidade imaginária a propriedade $i^{2}=-1$, sendo que $x$ é a parte real e $y$ parte imaginária do número complexo $z$. Segundo Bandim (2016), um lugar geométrico consiste no conjunto de pontos que gozam de uma específica propriedade matemática qualquer. Podem ser lugares geométricos curvas, superfícies e outras variedades quaisquer.

Uma aplicação que relaciona de modo unificado esses dois conteúdos é dada por Brown e Churchill (2009, p. 29) ao definir a região $\left|z-z_{0}\right|<\varepsilon$ no plano complexo como sendo a região de um disco, conforme Figura 1.

Figura 1 - Disco no plano complexo formado por $\left|z-z_{0}\right|<\mathcal{E}$

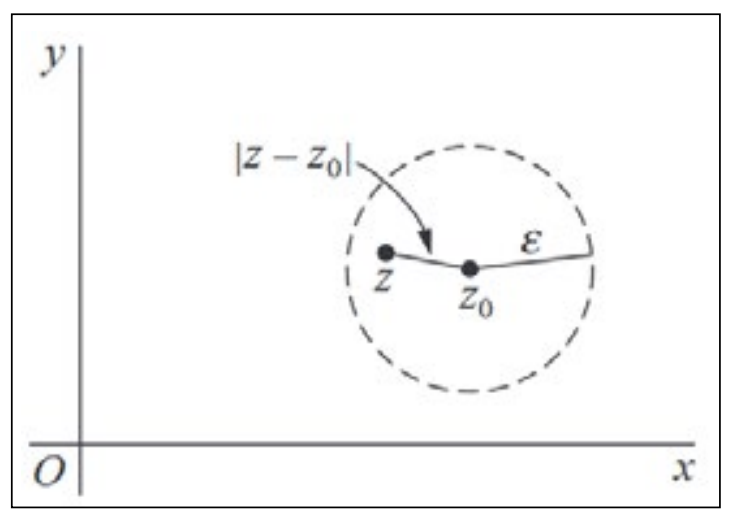

Fonte: Brown e Churchill (2009, p. 29).

Considerando a importância de realizar conexões entre conteúdos matemáticos e explorá-los, tendo a ideia de Brown e Churchill (2009, p. 29) como motivadora, este trabalho tem o objetivo de fazer relações entre os conteúdos de Números Complexos e Lugares Geométricos. Explorando a mediatriz, o círculo, a elipse, a hipérbole e a parábola no plano complexo. Segundo Gil (2010), este trabalho se enquadra como básico puro, por se dedicar exclusivamente à ampliação do conhecimento.

\section{LUGARES GEOMÉTRICOS NO PLANO COMPLEXO}

\subsection{Mediatriz}

Tendo a noção do que seja um lugar geométrico, analisemos o significado da equação $z=\left\{\left|z-z_{1}\right|=\left|z-z_{2}\right|: z_{1}, z_{2} \in \mathbb{C}\right\}$, podemos perceber que $\left|z-z_{1}\right| \mathrm{e}\left|z-z_{2}\right|$ são distâncias entre dois números complexos e que essas distâncias são iguais. Porém, o complexo $z$ não está definido. Podemos então concluir que a equação representa o conjunto de pontos que são equidistantes dos complexos $z_{1}$ e $z_{2}$, ou seja, a equação representa a mediatriz do segmento 
$\overline{z_{1} z_{2}}$ (conforme Figura 1). Verificando analiticamente, consideramos $z=x+y i$, $z_{1}=x_{1}+y_{1} i$ e $z_{2}=x_{2}+y_{2} i$ teremos

$$
\left|z-z_{1}\right|=\left|x+y i-x_{1}-y_{1} i\right|=\sqrt{\left(x-x_{1}\right)^{2}+\left(y-y_{1}\right)^{2}}
$$

e

$$
\left|z+z_{2}\right|=\left|x+y i-x_{2}-y_{2} i\right|=\sqrt{\left(x-x_{2}\right)^{2}+\left(y-y_{2}\right)^{2}}
$$

portanto fazendo a igualdade e alguns cálculos algébricos, temos

$$
\begin{aligned}
& \sqrt{\left(x-x_{1}\right)^{2}+\left(y-y_{1}\right)^{2}}=\sqrt{\left(x-x_{2}\right)^{2}+\left(y-y_{2}\right)^{2}} \\
& x^{2}-2 \cdot x \cdot x_{1}+x_{1}^{2}+y^{2}-2 \cdot y \cdot y_{1}+y_{1}^{2}=x^{2}-2 \cdot x \cdot x_{2}+x_{2}^{2}+y^{2}-2 \cdot y \cdot y_{2}+y_{2}^{2} \\
& -2 \cdot x \cdot x_{1}+2 \cdot x \cdot x_{2}-2 \cdot y \cdot y_{1}+2 \cdot y \cdot y_{2}=x_{2}^{2}-x_{1}^{2}+y_{2}^{2}-y_{1}^{2}
\end{aligned}
$$

Colocando alguns termos em evidência no primeiro membro e fatorando no segundo membro, tem-se

$$
\begin{aligned}
& \text { 2. } x\left(x_{2}-x_{1}\right)+2 \cdot y\left(y_{2}-y_{1}\right)=\left(x_{2}-x_{1}\right)\left(x_{2}+x_{1}\right)+\left(y_{2}-y_{1}\right)\left(y_{2}+y_{1}\right) \\
& \text { 2. } y\left(y_{2}-y_{1}\right)-\left(y_{2}-y_{1}\right)\left(y_{2}+y_{1}\right)=-2 \cdot x\left(x_{2}-x_{1}\right)+\left(x_{2}-x_{1}\right)\left(x_{2}+x_{1}\right) \\
& \left(2 y-y_{2}-y_{1}\right)\left(y_{2}-y_{1}\right)=-\left(2 x-x_{2}-x_{1}\right)\left(x_{2}-x_{1}\right) \\
& \left(2 y-y_{2}-y_{1}\right)=-\frac{\left(x_{2}-x_{1}\right)}{\left(y_{2}-y_{1}\right)}\left(2 x-x_{2}-x_{1}\right)
\end{aligned}
$$

Dividindo os dois lados da equação por dois, tem-se

$$
\left(y-\frac{y_{2}+y_{1}}{2}\right)=-\frac{\left(x_{2}-x_{1}\right)}{\left(y_{2}-y_{1}\right)}\left(x-\frac{x_{2}+x_{1}}{2}\right)
$$

que é a mediatriz do segmento $\overline{z_{1} z_{2}}$, como podemos observar na Figura 2.

Figura 2 - Representação da mediatriz dos pontos $z_{1}$ e $z_{2}$

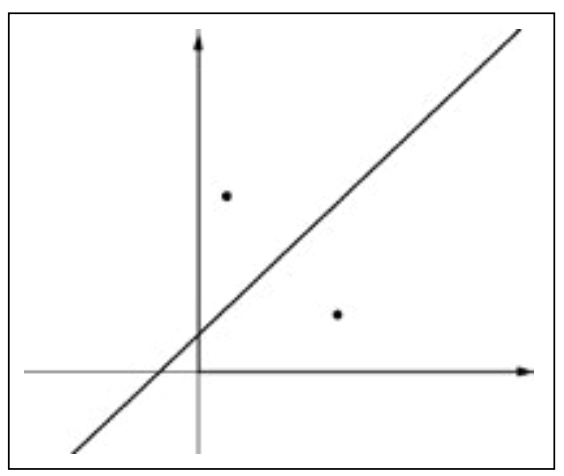

Fonte: Dos autores. 


\subsection{Circunferência}

Agora, fazendo uma análise do conjunto $z=\left\{\left|z-z_{1}\right|=r: z_{1} \in \mathbb{C}, r \mathbb{R}\right\}$, podemos perceber que $\left|z-z_{l}\right|$ é uma distância entre dois números complexos e o valor de $r$ é a medida da distância entre esses dois complexos. Porém, o complexo $z$ não está definido e o complexo $z_{1}$ está definido. Podemos então concluir que se refere à distância de um ponto fixo no plano complexo, que no caso é $z_{l}$, é sempre constante, que no caso é $r$. Portanto, podemos tirar a conclusão de que o lugar geométrico da equação $\left|z-z_{l}\right|=r$ representa uma circunferência de centro $z_{l}$ e raio $r$.

Para uma verificação analítica, consideremos os complexos $z=x+y i \mathrm{e}$ $z_{1}=x_{1}+y_{1} i$, desse modo teremos

$$
\left|z-z_{1}\right|=\left|x+y i-x_{-} 1-y_{1}\right||=|\left(x-x_{1}\right)+\left(y-y_{1}\right) i \mid=\sqrt{\left(x-x_{1}\right)^{2}+\left(y-y_{1}\right)^{2}}=T
$$

que é o mesmo que $\left(x-x_{1}\right)^{2}+\left(y-y_{1}\right)^{2}=r^{2}$. Que corresponde a uma equação de circunferência onde, como previmos, tem centro $z_{1}$ e raio $r$, que podemos conferir na Figura 3.

Figura 3 - Representação da circunferência de Centro $C$ e Raio $r$

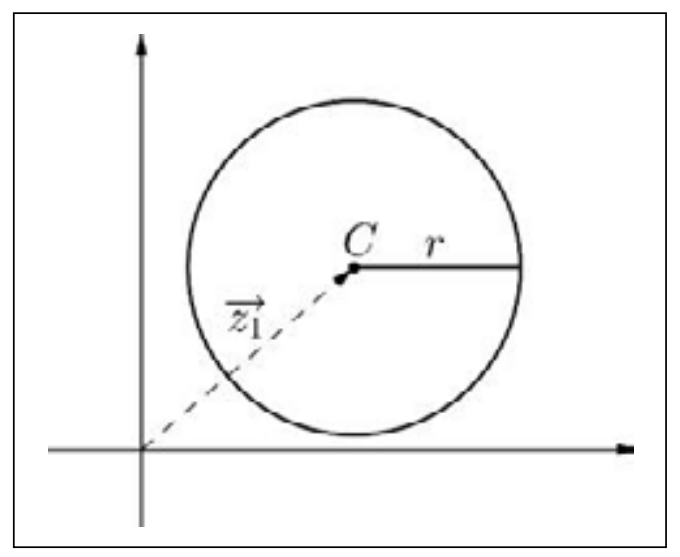

Fonte: Dos autores.

\subsection{Elipse}

Considere o conjunto dado por $z=\{|z-c|+|z+c|=2 a: c, a \in \mathbb{R}\}$ . Representa todos os pontos cuja soma de suas distâncias a dois pontos fixos é igual $2 a$. O lugar geométrico descrito é a definição de elipse com focos horizontais nos complexos $C$ e $-C$. Fazendo uma verificação analítica temos que, para $z=x+y i$ temos

$$
|z-c|+|z+c|=|x+y i-c|+|x+y i+c|=\sqrt{(x-c)^{2}+y^{2}}+\sqrt{(x+c)^{2}+y^{2}}=2 a
$$

que fazendo uma manipulação algébrica, obtemos 
$\sqrt{(x+c)^{2}+y^{2}}=2 a-\sqrt{(x-c)^{2}+y^{2}}$

elevando ambos os membros ao quadrado

$(x+c)^{2}+y^{2}=4 a^{2}-4 a \sqrt{(x-c)^{2}+y^{2}}+(x-c)^{2}+y^{2}$

resolvendo os quadrados externos à radiciação

$x^{2}+2 c x+c^{2}+y^{2}=4 a^{2}-4 a \sqrt{(x-c)^{2}+y^{2}}+x^{2}-2 c x+c^{2}+y^{2}$

simplificando os termos iguais e dividindo ambos os membros por 4

$2 c x=4 a^{2}-4 a \sqrt{(x-c)^{2}+y^{2}}-2 c x$

$2 c x+2 c x=4 a^{2}-4 a \sqrt{(x-c)^{2}+y^{2}}$

$a \sqrt{(x-c)^{2}+y^{2}}=a^{2}-c x$

elevando novamente os membros ao quadrado e fazendo algumas manipulações

$$
\begin{aligned}
& a^{2}\left((x-c)^{2}+y^{2}\right)=\left(a^{2}-c x\right)^{2} \\
& a^{2}\left(x^{2}-2 c x+c^{2}+y^{2}\right)=a^{4}-2 a^{2} c x+c^{2} x^{2} \\
& a^{2} x^{2}-2 a^{2} c x+a^{2} c^{2}+a^{2} y^{2}=a^{4}-2 a^{2} c x+c^{2} x^{2}
\end{aligned}
$$

convenientes

simplificando o termo $-2 a^{2} c x$ e fazendo manipulações algébricas

$$
\begin{aligned}
& a^{2} x^{2}+a^{2} c^{2}+a^{2} y^{2}=a^{4}+c^{2} x^{2} \\
& a^{2} x^{2}-c^{2} x^{2}+a^{2} y^{2}=a^{4}-a^{2} c^{2} \\
& x^{2}\left(a^{2}-c^{2}\right)+a^{2} y^{2}=a^{2}\left(a^{2}-c^{2}\right)
\end{aligned}
$$

do teorema de Pitágoras $a^{2}=b^{2}+c^{2} \rightarrow b^{2}=a^{2}-c^{2}$ e substituindo $b^{2} x^{2}+a^{2} y^{2}=a^{2} b^{2}$

dividindo ambos os termos por $a^{2} b^{2}$

$\frac{b^{2} x^{2}}{a^{2} b^{2}}+\frac{a^{2} y^{2}}{a^{2} b^{2}}=\frac{a^{2} b^{2}}{a^{2} b^{2}}$

finalmente chegamos à equação reduzida da elipse

$\frac{x^{2}}{a^{2}}+\frac{y^{2}}{b^{2}}=1$

Que podemos observar na Figura 4. 
Figura 4 - Representação da elipse de focos $(-c, 0)$ e $(c, 0)$

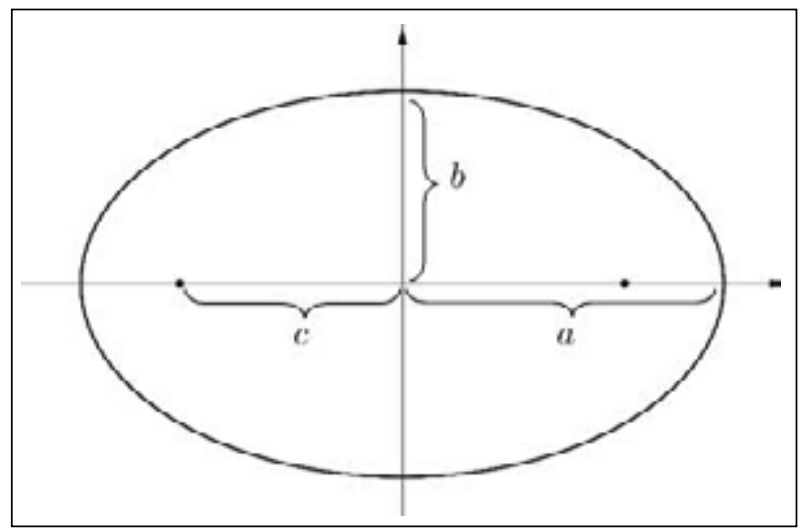

Fonte: Dos autores.

Para a equação reduzida da elipse com focos na vertical, temos de considerar o conjunto $z=\{|z-c i|+|z+c i|=2 a: c, a \in R\}$, considerando $z=x+y i$ temos

$$
\begin{aligned}
& |z-c i|+|z+c i|=|x+y i-c i|+|x+y i+c i| \\
& \sqrt{x^{2}+(y-c)^{2}}+\sqrt{x^{2}+(y+c)^{2}}=2 a
\end{aligned}
$$

que fazendo uma manipulação algébrica, obtemos

$$
\sqrt{x^{2}+(y+c)^{2}}=2 a-\sqrt{x^{2}+(y-c)^{2}}
$$

elevando ambos os membros ao quadrado

$$
x^{2}+(y+c)^{2}=4 a^{2}-4 a \sqrt{x^{2}+(y-c)^{2}}+x^{2}+(y-c)^{2}
$$

resolvendo os quadrados externos à radiciação

$$
x^{2}+y^{2}+2 c y+c^{2}=4 a^{2}-4 a \sqrt{x^{2}+(y-c)^{2}}+x^{2}+y^{2}-2 c y+c^{2}
$$

simplificando os termos iguais e dividindo ambos os membros por 4

$$
\begin{aligned}
& 2 c y=4 a^{2}-4 a \sqrt{x^{2}+(y-c)^{2}}-2 c y \\
& 2 c y+2 c y=4 a^{2}-4 a \sqrt{x^{2}+(y-c)^{2}} \\
& \sqrt{a x^{2}+(y-c)^{2}}=a^{2}-c y
\end{aligned}
$$

manipulações

elevando novamente os membros ao quadrado e fazendo algumas

$$
\begin{aligned}
& a^{2}\left(x^{2}+(y-c)^{2}\right)=\left(a^{2}-c y\right)^{2} \\
& a^{2}\left(x^{2}+y^{2}-2 c y+c^{2}\right)=a^{4}-2 a^{2} c y+c^{2} y^{2}
\end{aligned}
$$




$$
a^{2} x^{2}+a^{2} y^{2}-2 a^{2} c y+a^{2} c^{2}=a^{4}-2 a^{2} c y+c^{2} y^{2}
$$

simplificando o termo $-2 a^{2} c y$ e fazendo manipulações algébricas convenientes

$$
\begin{aligned}
& a^{2} y^{2}+a^{2} c^{2}+a^{2} x^{2}=a^{4}+c^{2} y^{2} \\
& a^{2} y^{2}-c^{2} y^{2}+a^{2} x^{2}=a^{4}-a^{2} c^{2} \\
& y^{2}\left(a^{2}-c^{2}\right)+a^{2} x^{2}=a^{2}\left(a^{2}-c^{2}\right)
\end{aligned}
$$

do teorema de Pitágoras $a^{2}=b^{2}+c^{2} \rightarrow b^{2}=a^{2}-c^{2}$ e substituindo $b^{2} y^{2}+a^{2} x^{2}=a^{2} b^{2}$

dividindo ambos os termos por $a^{2} b^{2}$

$\frac{b^{2} y^{2}}{a^{2} b^{2}}+\frac{a^{2} x^{2}}{a^{2} b^{2}}=\frac{a^{2} b^{2}}{a^{2} b^{2}}$

finalmente chegamos à equação reduzida da elipse

$\frac{y^{2}}{a^{2}}+\frac{x^{2}}{b^{2}}=1$

Que podemos observar na Figura 5.

Figura 5 - Representação da elipse de focos $(0,-c)$ e $(0, c)$

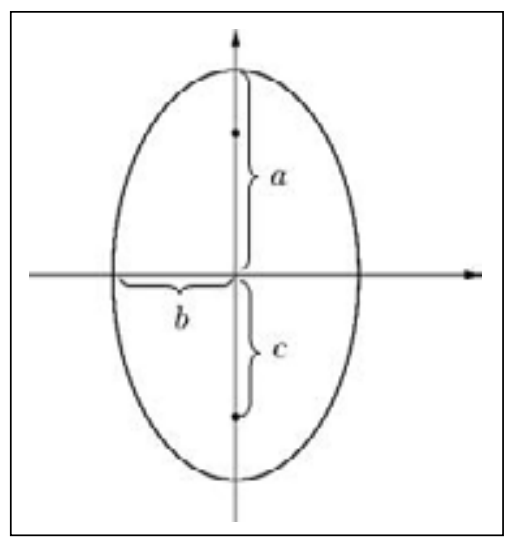

Fonte: Dos autores. 


\subsection{Hipérbole}

Nesse momento, considere o conjunto dado por

$$
z=|| z+c|-| z-c||= \pm 2 a: a, c \in \mathbb{R}
$$

que representa todos os pontos onde o módulo das diferenças de suas distâncias a dois pontos fixos é constante. O lugar geométrico descrito é a definição de hipérbole com focos nos complexos $-c$ e $c$. Para generalizarmos a equação da hipérbole com focos na horizontal, podemos considerar $z=x+y i$, então temos

$$
\begin{aligned}
& || z+c|-| z-c||=|| x+y i+c|-| x+y i-c||= \pm 2 a \\
& \sqrt{(x+c)^{2}+y^{2}}-\sqrt{(x-c)^{2}+y^{2}}= \pm 2 a \\
& \sqrt{(x+c)^{2}+y^{2}}=\sqrt{(x-c)^{2}+y^{2}} \pm 2 a
\end{aligned}
$$

elevando ambos os membros ao quadrado

$$
(x+c)^{2}+y^{2}=(x-c)^{2}+y^{2} \pm 4 a \sqrt{(x-c)^{2}+y^{2}}+4 a^{2}
$$

resolvendo as potenciações externas à radiciação e efetuando algumas manipulações

$$
\begin{aligned}
& x^{2}+2 c x+c^{2}+y^{2}=x^{2}-2 c x+c^{2}+y^{2} \pm 4 a \sqrt{(x-c)^{2}+y^{2}}+4 a^{2} \\
& 2 c x=-2 c x \pm 4 a \sqrt{(x-c)^{2}+y^{2}}+4 a^{2} \\
& 4 c x-4 a^{2}= \pm 4 a \sqrt{(x-c)^{2}+y^{2}}
\end{aligned}
$$

dividindo ambos os membros por 4

$$
c x-a^{2}= \pm a \sqrt{(x-c)^{2}+y^{2}}
$$

elevando novamente ambos os membros ao quadrado e posteriormente efetuando algumas manipulações algébricas

$$
\begin{aligned}
& \left(c x-a^{2}\right)^{2}=a^{2}\left((x-c)^{2}+y^{2}\right) \\
& \left(c x-a^{2}\right)^{2}=a^{2}\left(x^{2}-2 c x+c^{2}+y^{2}\right) \\
& c^{2} x^{2}-2 a^{2} c x+a^{4}=a^{2} x^{2}-2 a^{2} c x+a^{2} c^{2}+a^{2} y^{2}
\end{aligned}
$$

simplificando o termo $-2 a^{2} c x$ dos lados da equação

$$
\begin{aligned}
& c^{2} x^{2}+a^{4}=a^{2} x^{2}+a^{2} c^{2}+a^{2} y^{2} \\
& c^{2} x^{2}-a^{2} x^{2}-a^{2} y^{2}=a^{2} c^{2}-a^{4} \\
& x^{2}\left(c^{2}-a^{2}\right)-a^{2} y^{2}=a^{2}\left(c^{2}-a^{2}\right) \\
& x^{2} b^{2}-a^{2} y^{2}=a^{2} b^{2}
\end{aligned}
$$


dividindo a equação por $a^{2} b^{2}$

$\frac{x^{2} b^{2}}{a^{2} b^{2}}-\frac{a^{2} y^{2}}{a^{2} b^{2}}=\frac{a^{2} b^{2}}{a^{2} b^{2}}$

finalmente obtemos a equação reduzida da hipérbole com focos na horizontal

$$
\frac{x^{2}}{a^{2}}-\frac{y^{2}}{b^{2}}=1
$$

conforme a Figura 6.

Figura 6- Representação da hipérbole de focos $(-c, 0)$ e $(c, 0)$

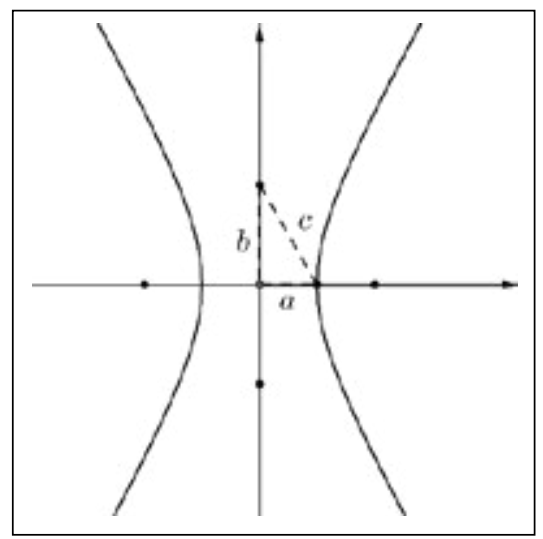

Fonte: Dos autores.

Para generalizarmos a equação da hipérbole com focos na vertical, consideraremos o conjunto $z=|| z+c i|-| z-c i||=2 a: c \in \mathbb{C}, a \in \mathbb{R}$ , também considerando $z=x+y i$ e procedendo de modo análogo (e mais resumido) teremos

$$
\begin{aligned}
& || z+c i|-| z-c i||=|| x+y i+c i|-| x+y i-c i||= \pm 2 a \\
& \sqrt{x^{2}+(y+c)^{2}}-\sqrt{x^{2}+(y-c)^{2}}= \pm 2 a \\
& \sqrt{x^{2}+(y+c)^{2}}= \pm 2 a+\sqrt{x^{2}+(y-c)^{2}}
\end{aligned}
$$

elevando aos membros da equação ao quadrado e fazendo algumas manipulações algébricas

$$
\begin{aligned}
& \left(c y-a^{2}\right)^{2}=a^{2}\left(x^{2}+(y-c)^{2}\right) \\
& \left(c y-a^{2}\right)^{2}=a^{2}\left(x^{2}+y^{2}-2 y x+c^{2}\right) \\
& c^{2} y^{2}+a^{4}=a^{2} x^{2}+a^{2} c^{2}+a^{2} y^{2}
\end{aligned}
$$




$$
\begin{aligned}
& c^{2} y^{2}-a^{2} y^{2}-a^{2} x^{2}=a^{2} c^{2}-a^{4} \\
& y^{2}\left(c^{2}-a^{2}\right)-a^{2} x^{2}=a^{2}\left(c^{2}-a^{2}\right) \\
& y^{2} b^{2}-a^{2} x^{2}=a^{2} b^{2}
\end{aligned}
$$

vertical

finalmente obtemos a equação reduzida da hipérbole com focos na

$$
\frac{y^{2}}{a^{2}}-\frac{x^{2}}{b^{2}}=1
$$

que podemos ver representado na Figura 7,

Figura 7 - Representação da hipérbole de focos $(0,-c)$ e $(0, c)$

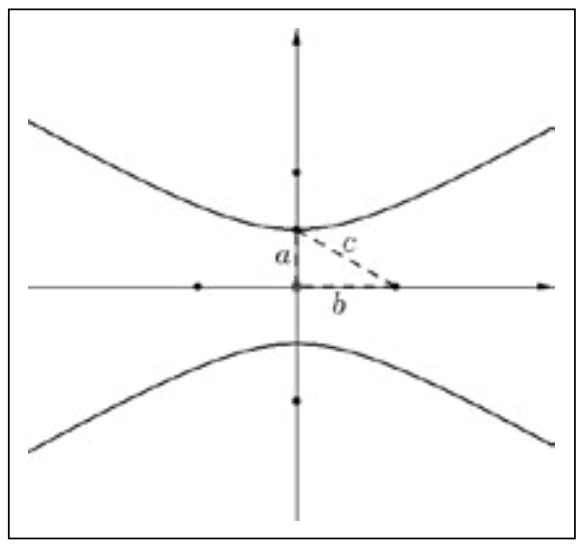

Fonte: Dos autores.

\subsection{Parábola}

Para finalizar as aplicações dos Números Complexos em lugares geométricos, veremos o sentido de $z=|z-a|=|\operatorname{Re}\{z\}+a|: a \in \mathbb{R}$, podemos perceber que $|z-a|$ é uma distância entre dois números complexos onde somente $a$ está definido. Antes do sentido de $|\operatorname{Re}\{z\}+a|$ ser verificado, podemos perceber que $\operatorname{Re}\{z\}$ representa uma reta vertical de abscissa igual à do complexo $z$ não definido, logo podemos perceber o sentido de $|\operatorname{Re}\{z\}+a|$ como sendo a medida da distância entre um complexo definido e uma reta vertical que denominamos de diretriz. Portanto podemos ter a conclusão que o lugar geométrico da equação $|z-a|=|\operatorname{Re}\{z\}+a|$ representa uma parábola com foco na horizontal e parâmetro $p=2 a$. Para uma verificação analítica, consideremos o complexo $z=x+y i$ desse modo teremos 


$$
\begin{aligned}
& |x+y i-a|=|x+a| \\
& \sqrt{(x-a)^{2}+y^{2}}=\sqrt{(x+a)^{2}}
\end{aligned}
$$

elevando os membros da equação ao quadrado e fazendo simples manipulações algébricas, temos

$$
\begin{aligned}
& (x-a)^{2}+y^{2}=(x+a)^{2} \\
& x^{2}-2 a x+a^{2}+y^{2}=x^{2}+2 a x+a^{2} \\
& -2 a x+y^{2}=2 a x
\end{aligned}
$$
horizontal

e, finalmente obtemos a equação reduzida da parábola com foco na

$$
\begin{aligned}
& y^{2}=4 a x \\
& \text { sendo } a=\frac{p}{2} \text {, onde } p \text { é o parâmetro da parábola, conforme a Figura } 8 .
\end{aligned}
$$

Figura 8 - Representação da parábola de Diretriz $d$ e Foco $(c, 0)$

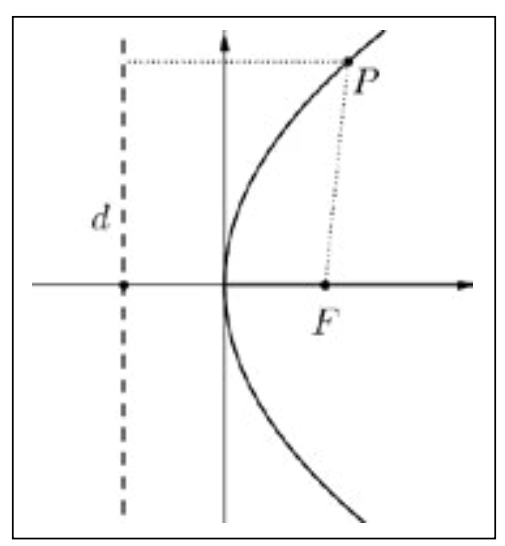

Fonte: Dos autores.

Para generalizarmos a equação da parábola com foco na vertical, basta considerar o conjunto dos pontos $z=|z-a i|=|\operatorname{Im}\{z\}+a i|: a i \in \mathbb{C}-\mathbb{R}$, também considerando $z=x+y i$ e procedendo de modo análogo, teremos

$$
\begin{aligned}
& |x+y i-a i|=|y i+a i| \\
& |x+(y-a) i|=|(y+a) i| \\
& \sqrt{x^{2}+(y-a)^{2}}=\sqrt{(y+a)^{2}}
\end{aligned}
$$

elevando os membros da equação ao quadrado e fazendo simples manipulações algébricas, temos 


$$
\begin{aligned}
& x^{2}+(y-a)^{2}=(y+a)^{2} \\
& -2 a y+x^{2}=2 a y
\end{aligned}
$$

vertical

e, finalmente obtemos a equação reduzida da parábola com foco na

$$
\begin{aligned}
& x^{2}=4 a y \\
& \text { sendo } a=\frac{p}{2} \text {, onde } p \text { é o parâmetro da parábola, conforme a Figura } 9 .
\end{aligned}
$$

Figura 9 - Representação da parábola de Diretriz $d$ e Foco $(0, c)$

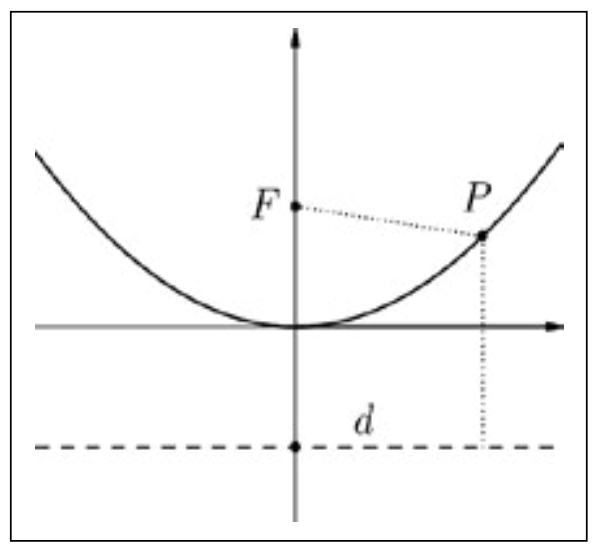

Fonte: Dos autores.

\section{CONSIDERAÇÕES FINAIS}

Neste trabalho destacamos a interconexão de conhecimentos matemáticos existentes entre Números Complexos e Lugar Geométricos. Sendo abordados os temas de mediatriz, círculo, elipse, hipérbole e parábola no plano complexo, explicitando as definições desses conhecimentos por meio de operações e expressões baseadas em um número complexo $z$, onde $z=x+y i$.

O tema de mediatrizes foi abordado explorando as duas distâncias $\left|z-z_{l}\right|$ e $\left|z-z_{2}\right|$ com o complexo $z$ não definido. Para a circunferência foi explanada a distância $\left|z-z_{l}\right|=r$ sendo o valor de $z_{l}$ e $r$ definidos e $z$ não definido. Sobre as cônicas elipse, hipérbole e parábola foram exploradas suas respectivas definições. Dessa forma, acreditamos ter ampliando as possibilidades de aplicações de Números Complexos dentro da própria matemática.

Para um estudo mais aprofundado sobre Números Complexos, recomendamos as obras de Ávila (2002) e Beirigo (2016). Os Números Complexos tiveram sua gênese por meio da fórmula de resolução de equações cúbicas, onde uma raiz quadrada de número negativo já não pôde ser tida 
como sem solução e hoje tem um campo vasto de aplicações na matemática e na física. Como em muitos conhecimentos matemáticos, uma grande descoberta pode ter uma humilde origem e parecer sem utilidade concreta e com os Números Complexos não foi diferente (LOPES, 2014). Por fim, destacamos uma conhecida frase de Nicolai Ivanovich Lobachevsky (1792-1856): “Não há nenhum ramo da Matemática, por mais abstrato que seja, que não possa um dia ser aplicado a fenômenos do mundo real" (STEIN, 2008, p. 29).

\section{REFERÊNCIAS}

ÁVILA, G. Variáveis complexas e aplicações. 3ª ed. Rio de Janeiro - RJ: SBM, 2002.

BANDIM, R. G. Lugar Geométrico - Uma abordagem com Geometria Dinâmica. Recife: Dissertação de Mestrado Profissional em Matemática - Universidade Federal Rural de Pernambudo (UFRPE), 2016.

BEIRIGO, T. Números complexos: uma metodologia baseada na história para obtenção de conceito. $1^{\mathrm{a}}$. ed. Joinvile: Clube de Autores, 2016.

BROWN, J. W.; CHURCHILL, R. V. Complex variables and applications. $8^{\text {a }}$. ed. New York: McGraw-Hill New York, 2009.

GIL, A. C. Como elaborar projetos de pesquisa. 5a . ed. São Paulo: Atlas, 2010.

LIMA, E. L. et al. A matemática do ensino médio. $4^{a}$. ed. Rio de Janeiro - RJ: SBM, v. 3, 2004.

LOPES, T. B. Uma metodologia baseada na história para obtenção de conceito sobre Números Complexos. Palmas - TO: Dissertação de Mestrado Profissional em Matemática - Universidade Federal do Tocantins (UFT), 2014. Disponivel em: <http:// bit.profmat-sbm.org.br/xmlui/bitstream/handle/123456789/1142/2012_00923_ THIAGO_BEIRIGO_LOPES.pdf >. Acesso em: 10 jun. 2015.

STEIN, J. Como a matemática explica o mundo: o poder dos números no cotidiano. Tradução de Marcio de Paula S. Hack. Rio de Janeiro: Elsevier, 2008. 